\title{
Modalités de prescription des injections intracaverneuses
}

\author{
Dominique DELAVIERRE
}

Service d'Urologie-Andrologie, CHR d'Orléans

RESUME

Les injections intracaverneuses sont indiquées dans le traitement de la dysfonction érectile en cas de contreindication, intolérance ou échec du traitement pharmacologique oral. Deux produits sont disponibles : le chlorhydrate de moxisylyte, facilitateur de l'érection nécessitant une stimulation sexuelle concomittante, et la prostaglandine PGE1 (alprostadii), inductrice de l'érection. Toute prescription est précédée d'une phase d'apprentissage de la technique et d'adaptation de la posologie. Le patient est informé du risque d'érection prolongée et de l'obligation de contacter le médecin prescripteur si une érection rigide se prolonge au-delà de 3 heures. Les principales contre-indications des injections intraca-verneuses sont les hémopathies pouvant donner des priapismes (drépanocytose, myélome multiple et leucémie) et les pathologies psychiatriques ne permettant pas une prescription sécurisée. Les injections intracaverneuses d'alprostadil sont remboursées par la sécurité sociale à $35 \%$ selon la procédure des médicaments d'exception et dans certaines situations uniquement.

Mots clés : dysfonction érectile, injections intracaverneuses, prostaglandines
Le principe thérapeutique des injections intracaverneuses est d'injecter dans un des corps caverneux une substance capable de provoquer une relaxation des fibres musculaires lisses et en conséquence une érection qualifiée de pharmaco-induite [3]. Les injections intracaverneuses sont indiquées dans le traitement de la dysfonction érectile en cas de contre-indication, intolérance ou échec du traitement pharmacologique oral [1].

1. Quels sont les produits disponibles ? $[1,2,3,4,5$, $6,7]$

- Le chlorhydrate de moxisylyte commercialisé depuis 1992 est disponible sous deux dosages 10 et $20 \mathrm{mg}$. C'est un facilitateur de l'érection et son utilisation nécessite une stimulation sexuelle concomitante.

- La prostaglandine PGE1 (alprostadil) commercialisée depuis 1994 est disponible également sous deux dosages 10 et $20 \mu \mathrm{g}$. II s'agit d'une substance inductrice de l'érection ne nécessitant pas de stimulation sexuelle.

\section{Comment pratiquer une injection intracaverneuse ?}

L'injection s'effectue sur la face latérale de la verge d'un côté ou de l'autre et non sur les faces dorsale (présence des paquets vasculo-nerveux dorsaux) ou ventrale (présence de l'urètre).

3. Comment prescrire les injections intracaverneuses ? $[1,2,3,6]$

La première injection s'effectue obligatoirement en milieu médical sous le contrôle d'un médecin disponible et entraîné. Toute prescription est précédée d'une phase d'apprentissage de la technique et d'adaptation de la

Correspondance :

Dr Dominique DELAVIERRE - Service d'Uro-Andrologie, CHR La Source, BP 6709, 45067 ORLEANS Cedex 2 - Tel 02.38.51.46.26 - Fax 02.38.51.41.59 - Email delav.uro@wanadoo.fr 
posologie par paliers avec pour objectif d'obtenir une érection d'une durée de 1 heure maximum. La prescription précise la nécessité pour le patient de respecter la posologie déterminée et de ne pas effectuer plus de 2 (alprostadil) ou 3 (moxisylyte) injections par semaine avec un délai de 24 heures minimum entre 2 injections. Le patient est informé du risque d'érection prolongée et de l'obligation de contacter le médecin prescripteur si une érection rigide se prolonge au-delà de 3 heures.

\section{Quelles sont les contre-indications des injections} intracaverneuses ? $[1,6]$ (Tableau 1 )

Tableau 1 : Contre-indications.

- Hémopathies pouvant donner des priapismes (drépanocytose, myélome multiple et leucémie) ;

- Pathologies psychiatriques ne permettant pas une prescription sécurisée :

- Pression artérielle systolique $<100 \mathrm{~mm} \mathrm{HG}$ (Moxisylyte).

- Hémopathies pouvant donner des priapismes (drépanocytose, myélome multiple et leucémie) [6] ;

- Pathologies psychiatriques ne permettant pas une prescription sécurisée [1] :

- Pression artérielle systolique $<100$ mm HG (Moxisylyte) [6]

5. Y a t'il des précautions dans certaines situations ? [6]

- Malformation anatomique du pénis (angulation, sclérose des corps caverneux et maladie de Lapeyronie) (risque de douleurs lors de l'érection);

- Troubles de coagulation et traitements anticoagulants (risque de saignement, ecchymose ou hématome au point d'injection) ;

- Association aux traitements antihypertenseurs (risque de potentialisation de l'effet antihypertenseur) ;

- Il est préférable d'éviter les injections intracaverneuses chez les patients ayant présenté un accident cardiovasculaire dans les 3 mois précédents et dont l'état cardiovasculaire n'est pas stabilisé ;

- Il est conseillé aux patients effectuant des injections intracaverneuses d'alprostadil et dont la partenaire est enceinte ou susceptible de l'être d'utiliser des préservatifs en raison du passage d'alprostadil dans le sperme et le liquide séminal ;

- Les injections intracaverneuses de moxisylyte sont déconseillées chez les patients traités par Alpha-bloquants (risque d'hypotension artérielle sévère).

6. Les injections intracaverneuses sont-elles remboursées par la sécurité sociale? [6] (Tableau 2)
Tableau 2 : Situations où les injections d'alprostadil sont remboursées.

- Paraplégie, tétraplégie :

- Traumatisme du bassin compliqué de troubles urinaires ;

- Séquelles de chirurgie (anévrysme de l'aorte, prostatectomie radicale, cystectomie totale, exérèse colo-rectale) ou de radiothérapie abdomino-pelvienne ;

- Séquelles de priapisme ;

- Neuropathie diabétique avérée :

- Sclérose en plaques

Les injections intracaverneuses d'alprostadil sont remboursées par la sécurité sociale à $35 \%$ selon la procédure des médicaments d'exception et dans certaines circonstances:

- Paraplégie, tétraplégie ;

- Traumatisme du bassin compliqué de troubles urinaires;

- Séquelles de chirurgie (anévrysme de l'aorte, prostatectomie radicale, cystectomie totale, exérèse colo-rectale) ou de radiothérapie abdomino-pelvienne ;

- Séquelles de priapisme ;

- Neuropathie diabétique avérée :

- Sclérose en plaques.

\section{CONCLUSION}

Les injections intracaverneuses appartiennent toujours à l'arsenal thérapeutique de la dysfonction érectile en raison notamment de leur efficacité [1], mais il s'agit d'un traitement de deuxième intention imposant certaines précautions et règles de prescription (Tableau 3).

Tableau 3 : Les différentes étapes de la prescription.

- Vérification de l'absence de contre-indications.

- Phase d'apprentissage de la technique et d'adaptation de la posologie par paliers en milieu

médical.

- Information du patient :

- Modalités d'utilisation ;

- Effets secondaires éventuels (notamment risque d'érection prolongée):

- Obligation de contacter le médecin prescripteur si une érection rigide se prolonge au delà de 3 heures;

- Précautions d'emploi.

- Remise et explication de l'ordonnance. 


\title{
REFERENCES
}

1. BONDIL P. : Injection intracaverneuse. In : Bondil P. ed. La dysfonction érectile. Paris, John Libbey Eurotext, 2003 : 160-169.

2. DELAVIERRE D., PENEAU M., IBRAHIM H.: Injections intra-caverneuses. Encycl. Med. Chir. (Elsevier, Paris), Néphrologie-Urologie, Fa 18-395-A-10, 1996, 3 p.

3. GIULIANO F. : Les traitements pharmacologiques de l'insuffisance érectile. Andrologie, 2002, 12 : 84-90.

4. LEDDAA. : Erectile dysfunction : intracavernous treatment. Curr. Mer. Res. Opin., 2000, 16 (suppl 1) : 59-62.

5. LEUNGWATTANAKIJ S., FLYNN V. jr, HELLSTROM W.J. : Intracavernosal injection and intraurethral therapy for erectile dysfunction. Urol. Clin. North Am., 2001, 28 : 343-354.

6. RCP (Résumé des Caractèristiques du Produit) VIDAL 2004.

7. VIRAG R., BECHER E., CARRIER S. et al. : Local pharmacological treatment modalities. In : Jardin A., Wagner G., Khoury S. et al. eds. Erectile dysfunction. Health publication Ltd, 2000. 307-341.

\author{
ABSTRACT \\ Modalities of prescription intracavernous injections
}

\section{Dominique DELAVIERRE}

Intracavernous injections are indicated in the treatment of erectile dysfunction in patients presenting a contraindication, intolerance or failure of oral drug therapy. Two products are available: moxisylyte hydrochloride, facilitating erection and requiring concomitant sexual stimulation, and prostaglandin PGE1 (alprostadil), inducing erection. All prescriptions must be preceded by a learning phase of the technique and adaptation of the dosage. The patient is informed about the risk of prolonged erection and the need to contact the prescribing doctor if rigid erection persists for more than 3 hours. The main contraindications to intracavernous injection are haematological malignancies that can cause priapism (sickle-cell anaemia, multiple myeloma and leukaemia) and psychiatric diseases not allowing safe prescription. Intracavernous injections of alprostadil are $35 \%$ reimbursed by French social security according to the exceptional drug procedure and only in certain situations.

Key-Words: erectile dysfunction, intracavernous injections, prostaglandins 